Review

\title{
Recent Advancement in Optical Fiber Sensing for Aerospace Composite Structures
}

\author{
Shu MINAKUCHI* and Nobuo TAKEDA \\ Graduate School of Frontier Sciences, The University of Tokyo, 5-1-5 Kashiwanoha, Kashiwa-shi, Chiba 277-8561, \\ Japan \\ *Corresponding author: Shu MINAKUCHI～E-mail: minakuchi@smart.k.u-tokyo.ac.jp
}

\begin{abstract}
Optical fiber sensors have attracted considerable attention in health monitoring of aerospace composite structures. This paper briefly reviews our recent advancement mainly in Brillouin-based distributed sensing. Damage detection, life cycle monitoring and shape reconstruction systems applicable to large-scale composite structures are presented, and new technical concepts, "smart crack arrester" and "hierarchical sensing system", are described as well, highlighting the great potential of optical fiber sensors for the structural health monitoring (SHM) field.
\end{abstract}

Keywords: Carbon fiber reinforced plastic (CFRP), Brillouin-based system, fiber Bragg grating (FBG)

Citation: Shu MINAKUCHI and Nobuo TAKEDA, "Recent Advancement in Optical Fiber Sensing for Aerospace Composite Structures," Photonic Sensors, DOI: 10.1007/s13320-013-0133-4.

\section{Introduction}

Even though the carbon fiber reinforced plastic (CFRP) is being used in almost all modern aerospace structures as a primary structural material, it is still difficult to precisely manufacture largescale CFRP structures and ensure their structural integrity during operation. Hence, there is an urgent need to develop innovative techniques to monitor the internal states of composite structures and utilize the obtained data to improve the structural design, processing technologies and maintenance methods. In this context, several structural health monitoring (SHM) systems have been proposed and evaluated [1]. Among the developed systems, optical fiber sensors have attracted considerable attention [2-4], since they are small, lightweight, immune to electromagnetic interference, and environmentally stable, and they have very little signal loss over extremely long distances. Furthermore, they possess sufficient flexibility, strength, and heat resistance to be embedded relatively easily into composite laminates.

Our previous paper, published in 2007 [5], presented the development of small-diameter optical fibers and their fiber Bragg grating (FBG) sensors. The paper showed several techniques for detecting composite damage and gave a summary of the Japanese national project on optical-fiber-based SHM for feasible applications in aerospace composite structures. Following the previous paper, this paper briefly reviews our recent advancement mainly in Brillouin-based distributed sensing. Several techniques applicable to large-scale

Received: 19 July 2013 / Revised version: 31 August 2013

(C) The Author(s) 2013. This article is published with open access at Springerlink.com 
composite structures are presented, and additionally, new technical concepts, "smart crack arrester" and "hierarchical sensing system", are described.

\section{Damage detection using Brillouin based distributed strain sensing}

\subsection{Damage monitoring of CFRP bolted joints using strain distribution}

Bolted joints are structural key elements in current composite aircraft structures [6]. There is an urgent need to develop a new technique for detecting bearing failures in their initial stage since these failures can lead to catastrophic structural damage, but are invisible from the outside [6-8]. The authors developed a fiber-optic-based system applicable to large-scale composite structures having a massive number of the bolted joints [9]. Optical fibers were embedded along bolt holes, and the strain change along the optical fiber induced by bearing failure was measured by a Brillouin optical correlation domain analysis (BOCDA), which is a high spatial resolution distributed strain sensing system developed by Hotate et al. [10-12].

This study began by investigating damage modes of the CFRP bolted joints after bearing failure [13]. Effective embedding positions of optical fibers were then proposed, and their feasibility was evaluated by finite element analysis simulating the damage propagation in the bolted joint and consequent residual strain change [14]. Finally, verification tests were conducted using specimens with embedded polyimide-coated optical fibers at various positions. The results are summarized in Figs. 1 and 2. It was clearly shown that the damage could be detected using the residual strain due to fiber-microbuckling (kinking) damage or permanent deformation of the neighboring plies. Furthermore, the damage size and direction could be estimated from the change in the strain distribution. The system developed is quite useful for a first inspection of large-scale composite structures.

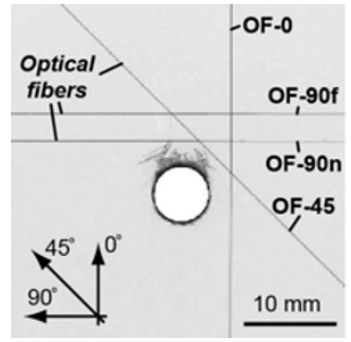

(a) After 1st loading

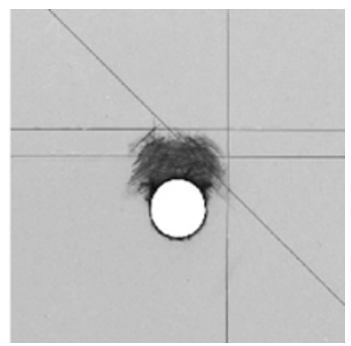

(b) After 2nd loading
Fig. 1 Soft X-ray images of damage around the CFRP bolt hole [9].
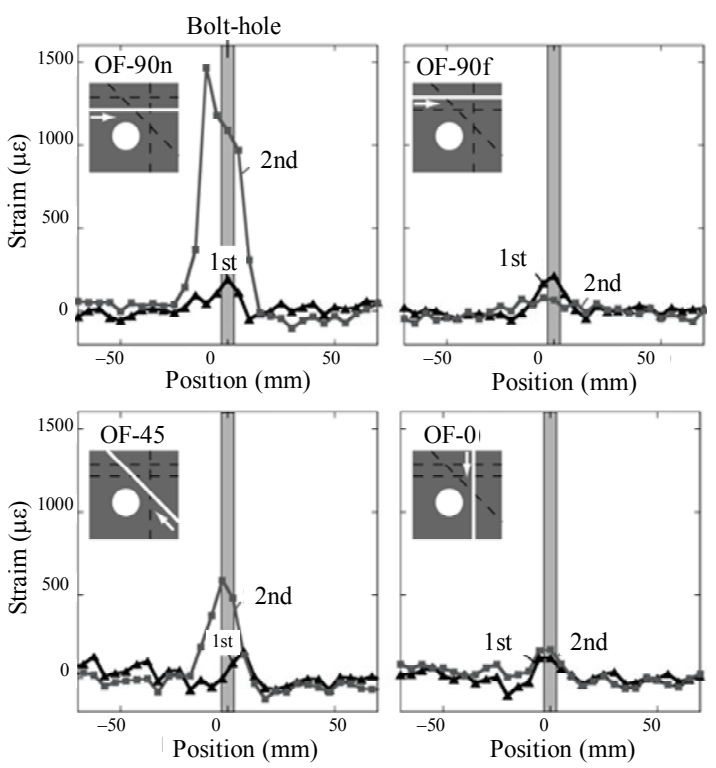

Fig. 2 Residual strain distribution along embedded optical fibers after each loading [9].

2.2 Damage monitoring of sandwich structures using Brillouin spectral response to non-uniform strain

In general, the central frequency of a measured Brillouin gain spectrum (BGS) represents the axial strain averaged over the spatial resolution of the measurement system. Thus, the strain change induced by damage smaller than the spatial resolution hardly changes the BGS central frequency. However, the shape of the BGS has information on the strain distribution within the spatial resolution $[15,16]$ and thus can be more sensitive to the damage. The authors investigated the effects of the strain profile within the spatial resolution on the 
BGS response [17] and demonstrated that the BGS becomes broader due to the non-uniform strain along the optical fiber. When a uniform strain is applied, the Brillouin frequency is also uniform, and thus the BGS has only one sharp narrow peak [Fig. 3 (a)]. When a non-uniform strain is introduced, however, the Brillouin frequency also becomes non-uniform, since the Brillouin frequency at each position on the optical fiber is determined by the strain at that position. As a result, the BGS consisting of the total Brillouin scattering within the spatial resolution becomes broad, as illustrated in Fig. 3(b). The authors utilized this phenomenon to detect impact damage in composite sandwich structures [18]. Pre-pump pulse Brillouin optical time-domain analysis (PPP-BOTDA) was utilized for distributed strain measurement $[19,20]$.

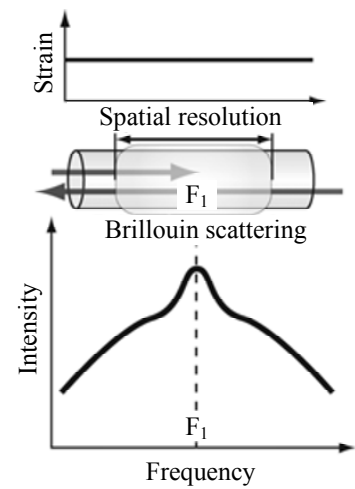

(a) Uniform strain

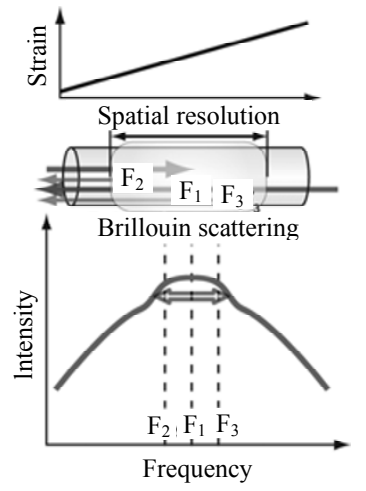

(b) Non-uniform strain
Fig. 3 Brillouin gain spectrum depending on the strain profile within the spatial resolution [18].

Composite sandwich structures are integral constructions consisting of two composite facesheets and a lightweight core, which can maximize the potentials of composite materials [21]. However, when the impact or indentation loading is applied, the core under the loading point crushes, and a residual dent remains on the facesheet, which significantly degrades the strength of the sandwich structure [22]. A schematic of the proposed impact damage detection system is illustrated in Fig. 4. The optical fibers are embedded in the adhesive layer between the facesheet and the core in a reticular pattern [23], measuring the strain along the bottom surface of the composite facesheet. The facesheet dent induces tensile and compressive strain along the embedded optical fiber at its concave and convex parts. This non-uniform strain changes the shape of the BGS, and thus the impact damage can be detected.

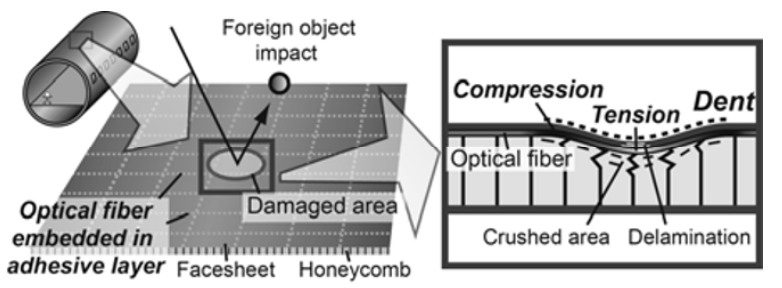

Fig. 4 Schematic of the impact damage detection system [18].

Firstly, the authors numerically simulated the response of the optical fiber sensor network to clarify the effectiveness and limitations of the proposed damage-detection technique. The proposed system was then validated by an experiment using a composite sandwich panel. Figure 5 presents the

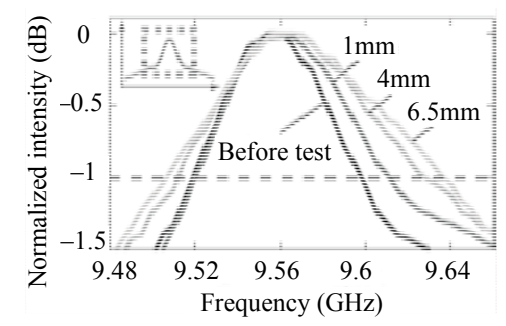

Fig. 5 BGS obtained from the measure point nearest to the loading point after each indentation loading [18].

spectra obtained from the measurement point nearest to the loading point. As the damage became larger, the width of the BGS gradually increased. In contrast, the peak frequency of the BGS hardly changed. This means that the distributed strain measurement using the peak frequency could not detect the damage. One example of two-dimensional distributions of the spectrum width is presented in Fig. 6. In this study, a full width at $-1 \mathrm{~dB}$ from the maximum $F_{-1 \mathrm{~dB}}$ was selected as a representative value for the width of the BGS, and each line represents the $F_{-1 \mathrm{~dB}}$ distribution along each line of 
the embedded optical fiber. As the damage became larger, the width of the Brillouin gain spectra became broader. Consequently, the location and size of the barely visible damage could be intuitively estimated. The BGS shape based technique was further utilized to detect water accumulation in honeycomb sandwich structures [24].

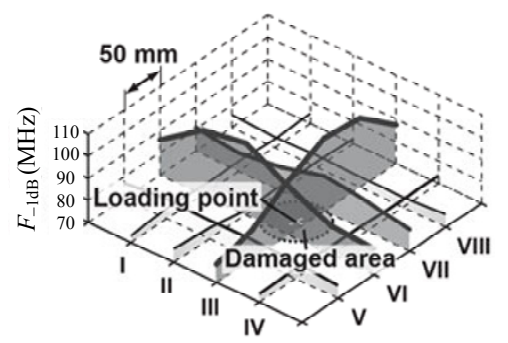

Fig. 6 Two-dimensional distribution of $F_{-1 \mathrm{~dB}}$ near the loading point after the indentation loading of $3 \mathrm{~mm}$ [18].

\section{Smart crack arrester with crack memorizing and detecting capability}

In composite sandwich structures, a crack below the facesheet is difficult to be detected using conventional non-destructive inspection techniques. However, an interface crack seriously degrades the structural integrity [21]. Thus, Hirose et al. developed a crack arrester (Fig. 7) [25], which is a semi-cylindrical stiff material inserted into the interface. When a crack approaches the arrester, the arrester decreases the energy release rate at the crack tip by suppressing local deformation around the crack. In practical applications, the arrester is arranged in a grid pattern, and the interface crack is trapped inside the grid (Fig. 7). The arrested crack should be detected instantaneously, and appropriate measures must be taken for the damaged area.

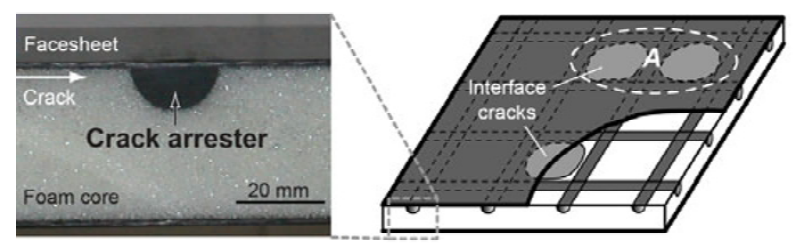

Fig. 7 Crack arrester [26].

The authors established the "smart crack arrester" that arrests, memorizes and detects interface crack propagation [26]. Figure 8 presents the schematic of the crack-detection technique. Two FBG sensors and two metal wires are embedded at both edges of the arrester. The characteristic strain state induced by arresting the high-speed crack propagation is first "memorized" by plastic deformation of the metal wire, and the consequent residual strain is then "statically" picked up as a damage signal by using a birefringence effect of the FBG sensor $[27,28]$. The system does not require a high-cost dynamic measurement system and can reliably detect the interface crack in a practical noisy environment.

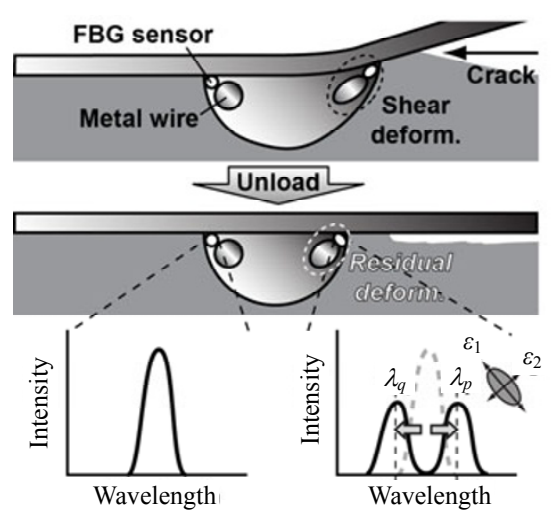

Fig. 8 Schematic of the crack-detection technique [it is important to note that metal wires added can be very thin (e.g., diameter less than $1 \mathrm{~mm}$ ), and thus a weight increase is negligible] [26].

The authors began by numerically simulating the deformation of the metal wires and the sensors to evaluate the feasibility of the proposed technique. The significant advantage of adding the metal wires was then demonstrated by comparing data from the proposed technique and the previous approach without metal wires [29]. Finally, a verification test was conducted to confirm that an FBG spectral shape statically obtained after the unloading can indicate the propagation direction and tip location of an arrested crack.

\section{Life cycle monitoring of the practical composite panel}

When optical fiber sensors are embedded into 
composite materials during manufacturing, the sensor can be used to continuously monitor the manufacturing process itself, in-service usage and damage. By combining all the information obtained by the distributed sensing network, we can accurately evaluate the structural health (Fig.9). The authors demonstrated life cycle monitoring of a representative CFRP stiffened panel manufactured by vacuum assisted resin transfer molding (VARTM) [30].

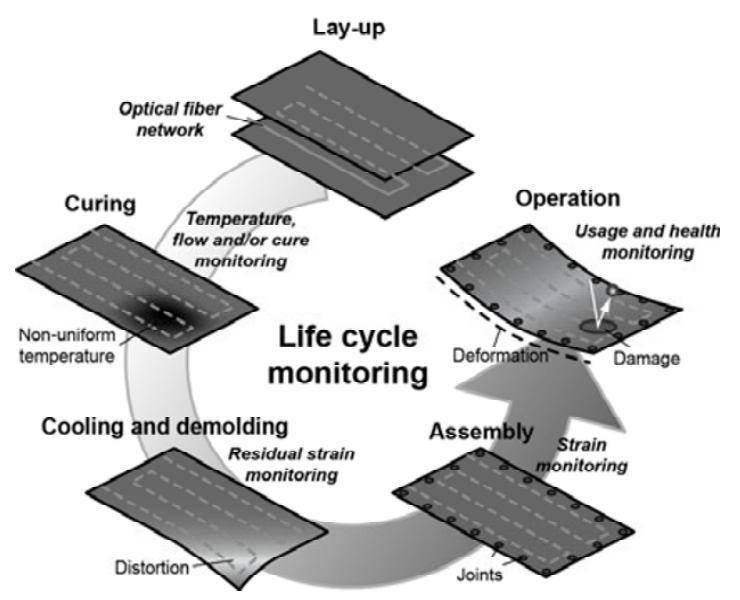

Fig. 9 Life cycle monitoring. [30].

Figure 10 depicts the schematic of the specimen. A single optical fiber was embedded between the stiffeners and the skin during the laminate lay-up process. In order to confirm the repeatability of measurement, the optical fiber went two circuits in the panel, monitoring the same place with two lines of the single optical fiber. PPP-BOTDA was used for the distributed sensing. Figure 11 shows the obtained residual strain distributions after manufacturing from the two lines of the embedded optical fiber. For better comparison, the horizontal axis is expressed in the distance from one end of the panel. The two lines measured quite similar strain distributions, confirming the high repeatability and reliability of the fiber-optic-based process monitoring. Almost the uniform compressive strain of $250 \mu \varepsilon$ was induced in the whole structural area, indicating that the specimen was perfectly injected and cured. The results also agreed well with the measurement by FBG sensors, validating the measurement accuracy of the distributed sensing. Subsequently, the manufactured panel was assembled, and lowvelocity impact loadings were applied. The fiberoptic network successfully monitored the strain change during the assembly and also detected two damages located near each other. After the impact tests, the embedded optical fiber still worked correctly, and the mechanical strain distribution around the damaged area could be obtained. The test result confirmed the effectiveness of life cycle monitoring by fiber-optic-based distributed sensing for developing highly-reliable composite structures.

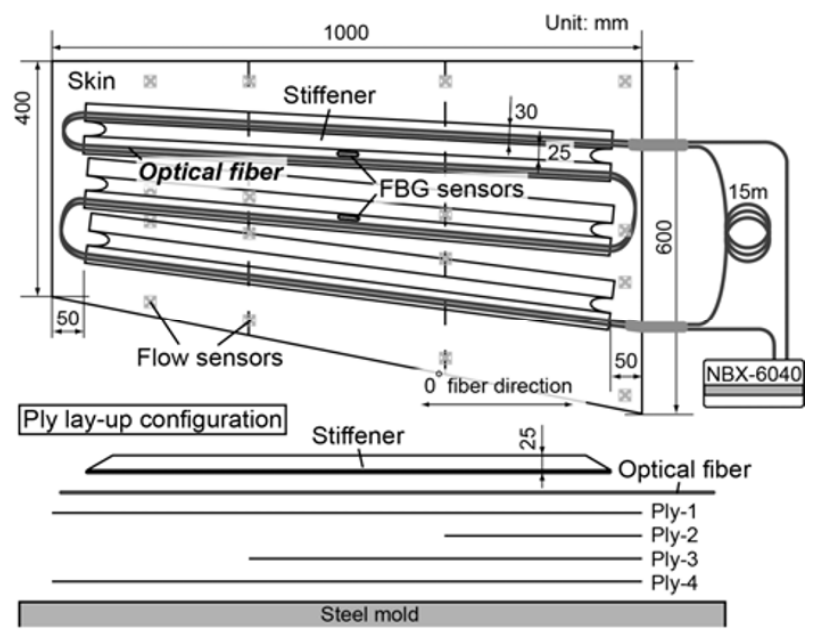

Fig. 10 Schematic of the representative CFRP stiffened panel specimen [30].

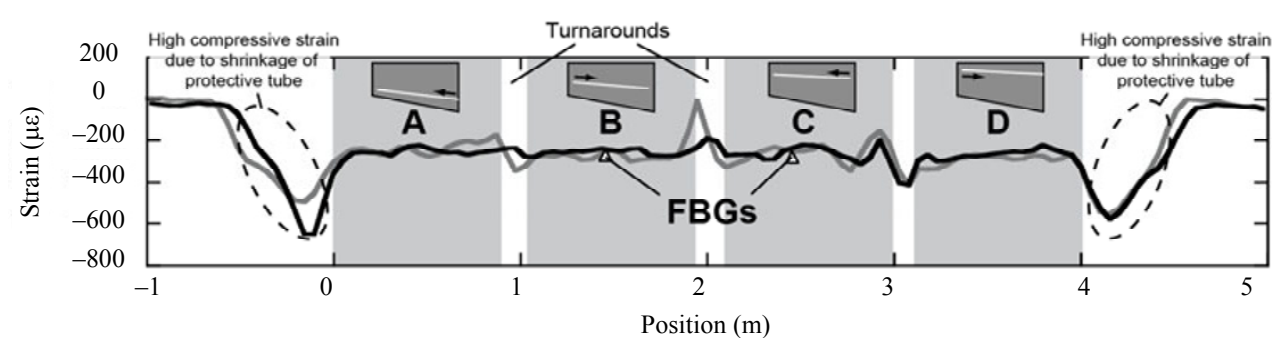

Fig. 11 Distribution of the thermal residual strain obtained from two lines embedded in the same position [30]. 
This life cycle monitoring concept was further demonstrated in complex-shaped parts [31, 32], and the advanced quality control method based on sensor responses was proposed. A hybrid BrillouinRayleigh system that can separately measure the strain and temperature distribution was also utilized for composite process monitoring [33].

\section{Full-field shape reconstruction using distributed strain data}

Shape reconstruction systems, which derive displacement from strain data, are especially suitable for understanding the global conditions of structures. Once an optical fiber network is embedded in the whole body of a structure, the shape reconstruction system can be applied not only to real-time deformation monitoring in the operation time, but also to distortion monitoring in the manufacturing process of composites. However, most previous work about shape reconstruction focused on only simple test structures since with conventional measurement systems (e.g., strain gauges and FBG sensors) the number or location of sensing points was limited [34, 35].

Our research group constructed a shape reconstruction algorithm using a finite element (FE) model of the target structure, taking advantage of characteristics of the distributed strain data obtained by PPP-BOTDA [36, 37]. The remarkable point is that, using not only raw strain data but also information of the non-uniformity of strain distribution profiles, the algorithm appropriately considers the data reliability for accurate shape reconstruction. The constructed algorithm was applied to the reconstruction of the deflection of a composite laminate specimen, in which an optical fiber network was embedded (Fig. 12). The results indicated that reconstruction accuracy was greatly improved by using weight values determined from the non-uniformity index of the strain distribution profile for each data point (Fig. 13). Moreover, the authors demonstrated that the prior information of identification parameters, which were the node-displacements of the FE model, could be provided for accurate shape reconstruction of complex deformation using the non-uniformity index.

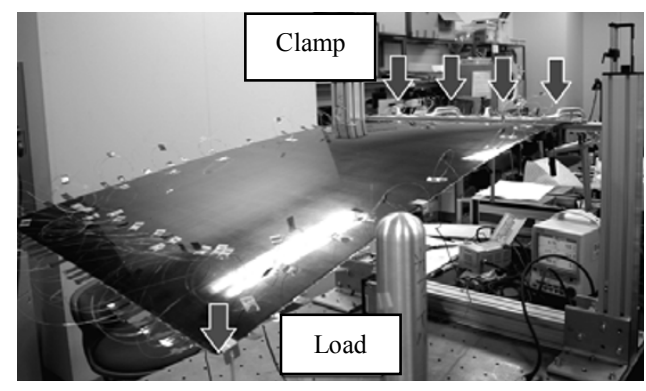

Fig. 12 Setup of cantilever plate bending test [37].

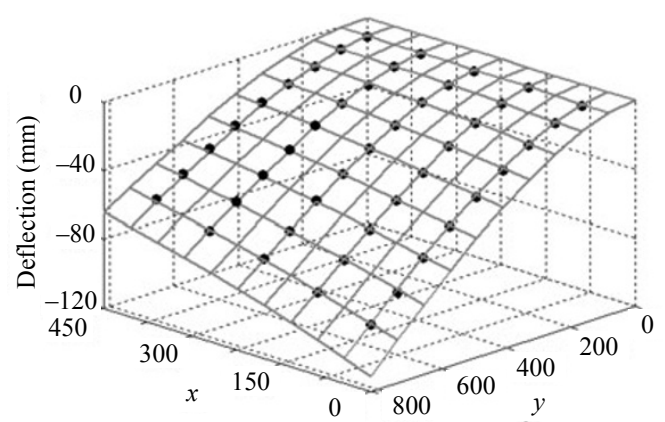

Fig. 13 Reconstructed deflection of the composite laminate specimen: the real points in the figure are the measured deflections using the laser displacement meter, and the reconstructed deflection shows good agreement with the measured deflection [37].

\section{Hierarchical sensing system with high monitorable area, robustness, and repairability}

When conventional fiber-optic-based systems [Fig. 14 (a)] are applied to large-scale structures for monitoring the randomly induced damage such as the impact damage [22], they are unsatisfactory in the following three properties: robustness, repairability, and monitorable area. Specifically, a failure at only one point on a sensing optical fiber leads to a breakdown of the entire sensing network. Moreover, once the optical fiber is disconnected, the damaged part needs to be repaired. However, it is quite difficult to access and reconnect the damaged 
fiber, especially when embedded. Furthermore, the fiber-optic sensing obtains basically a onedimensional strain (temperature) distribution along the thin fiber. Hence, the damage far from the sensing fiber cannot be detected, since it induces no significant strain (temperature) change in the fiber. To overcome these drawbacks, the authors proposed a hierarchical fiber-optic-based sensing system analogous to the nervous system in vertebrates [38]. In the hierarchical system [Fig. 14 (b)], several kinds of specialized devices are hierarchically combined to form a sensing network. Specifically, numerous three-dimensionally structured sensor devices are distributed throughout the whole structural area and connected with an optical fiber network (which is not embedded into the structure) through transducing mechanisms. The distributed "sensory nerve cell" devices detect the damage, and the fiberoptic "spinal cord" network gathers the damage signals and transmits the information to a measuring instrument. Since the optical fiber is attached to the back surface of the structure, the fiber rarely breaks when the structure is damaged. If by any chance the optical fiber is broken, the disconnected parts can be readily reconnected using a fusion splicer, since the optical fiber is relatively easily accessible. Finally, in the hierarchical system, several sensor devices connected to different optical fibers are placed in the same area; in Fig. 14 (b), two independent comb-like sensor devices share the same monitoring area. Hence, a failure at just one point in the devices or the fiber optic network does not affect the monitoring performance, and therefore, the hierarchical system has high redundancy and robustness.

In order to validate the hierarchical concept, a hierarchal impact damage detection system was developed with surface-crack sensing devices based on comparative vacuum monitoring (CVM), which was originally developed by Structural Monitoring Systems Ltd. in Australia [39-41]. The surfacemounted sensing devices are initially vacuumed, and an optical fiber network monitors the internal pressure in all of the deployed devices. Since the impact damage increases the internal pressure of the vacuumed device in the damaged area, one can identify the sensor device where the pressure change occurs and thus locate the damaged area. The proposed impact damage detection system was applied to a CFRP skin-stringer fuselage demonstrator (Fig. 15). The barely visible impact damage (BVID) was successfully detected from a strain increase in the optical fiber in the damaged area, and it was confirmed that the hierarchical system has the better repairability, higher robustness, and a wider monitorable area compared to existing fiber-optic-based systems.

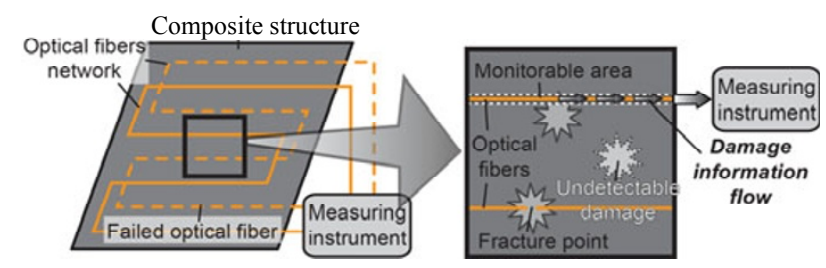

(a) Existing sensing system utilizing optical fiber network

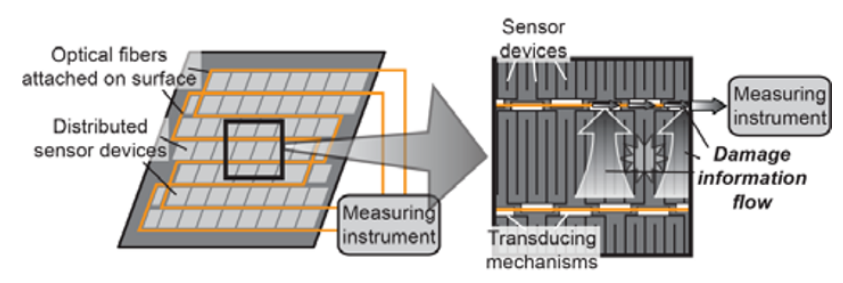

(b) Hierarchical sensing system combining distributed sensor devices and optical fibers

Fig. 14 Schematic of the sensing system [38].

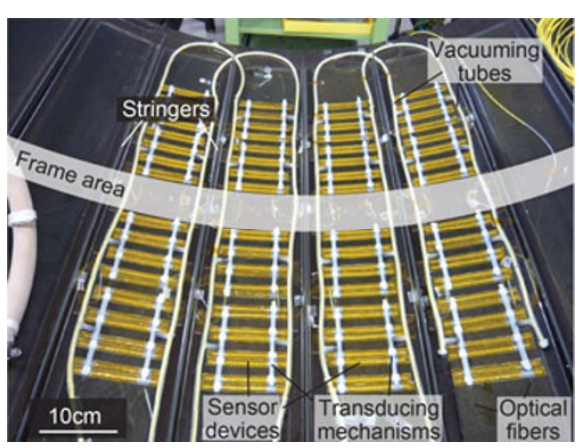

Fig. 15 Hierarchical surface-crack detection system deployed in the CFRP fuselage structure [38].

Recently, the sensitivity and durability of the hierarchical system were significantly improved by 
developing embeddable CVM sensor devices [42]. A further advance to be combined with a self-healing concept was also demonstrated.

\section{Conclusions}

This paper briefly reviewed our recent advancement mainly in Brillouin-based distributed sensing. Several systems applicable to large-scale composite structures were presented, and new technical concepts, "smart crack arrester" and "hierarchical sensing system", were described as well, highlighting the great potential for optical fiber sensors in the SHM field.

Open Access This article is distributed under the terms of the Creative Commons Attribution License which permits any use, distribution, and reproduction in any medium, provided the original author(s) and source are credited.

\section{References}

[1] C. Boller, F. K. Chang, and Y. Fujino, Encyclopedia of structural health monitoring. Chichester, UK: Wiley, 2009.

[2] K. S. C. Kuang and W. J. Cantwell, "Use of conventional optical fibers and fiber Bragg gratings for damage detection in advanced composite structures: a review," Applied Mechanics Reviews, vol. 56, no. 5, pp. 493-513, 2003.

[3] G. F. Fernando, "Fibre optic sensor systems for monitoring composite structures," Reinforced Plastics, vol. 49, no. 11, pp. 41-49, 2005.

[4] G. Luyckx, E. Voet, N. Lammens, and J. Degrieck, "Strain measurements of composite laminates with embedded fiber Bragg gratings: criticism and opportunities for research," Sensors, vol. 11, no. 1, pp. 384-408, 2011.

[5] N. Takeda, Y. Okabe, and T. Mizutani, "Damage detection in composites using optical fibre sensors," Proceedings of the Institution of Mechanical Engineers Part G: Journal of Aerospace Engineering, vol. 221, no. 4, pp. 497-508, 2007.

[6] S. D. Thoppul, J. Finegan, and R. F. Gibson, "Mechanics of mechanically fastened joints in polymer-matrix composite structures - a review,"
Composites Science and Technology, vol. 69, no. 3-4, pp. 301-329, 2009.

[7] Y. Xiao and T. Ishikawa, "Bearing strength and failure behavior of bolted composite joints (part i: experimental investigation)," Composites Science and Technology, vol. 65, no. 7-8, pp. 1022-1031, 2005.

[8] Y. Sato, T. Utsunomiya, T. Takatoya, and I. Susuki, "Application of smart bolt to early stage damage monitoring of composite shear fastener joint," Transactions of the Japan Society of Mechanical Engineers A, vol. 74, no. 737, pp. 90-96, 2008.

[9] S. Minakuchi, T. Nakamura, T. Nadabe, M. Nishikawa, N. Takeda, M. Kishi, et al., "Damage detection of CFRP bolted joints using embedded optical fibers with BOCDA system," Journal of the Japan Society for Aeronautical and Space Science, vol. 59, no. 690, pp. 176-182, 2011.

[10] K. Hotate and T. Hasegawa, "Measurement of Brillouin gain spectrum distribution along an optical fiber using a correlation-based technique proposal, experiment and simulation," IEICE Transactions on Electronics, vol. E83-C, no.3, pp. 405-412, 2000.

[11] K. Y. Song, Z. He, and K. Hotate, "Distributed strain measurement with millimeter-order spatial resolution based on Brillouin optical correlation domain analysis," Optics Letters, vol. 31, no. 17, pp. 2526-2529, 2006.

[12] K. Y. Song and K. Hotate, "Distributed fiber strain sensor with $1-\mathrm{kHz}$ sampling rate based on Brillouin optical correlation domain analysis," IEEE Photonics Technology Letters, vol. 19, no. 23, pp. 1928-1930, 2007.

[13] T. Nadabe, M. Nishikawa, S. Minakuchi, T. Nakamura, and N. Takeda, "Modeling of fiber kinking damage for bearing failure in bolted joints of CFRP laminates," Journal of the Japan Society for Composite Materials, vol. 37, no. 5, pp. 172-181, 2011.

[14] T. Nadabe, M. Nishikawa, S. Minakuchi, T. Nakamura, J. T. Siivola, and N. Takeda, "Numerical analysis for damage detection in CFRP bolted joints using strain measurement," Journal of the Japan Society for Composite Materials, vol. 38, no. 1, pp. 22-29, 2012.

[15] T. Horiguchi, T. Kurashima, M. Tateda, K. Ishihara, and Y. Wakui, "Brillouin characterization of fiber strain in bent slot-type optical-fiber cables," Journal of Lightwave Technology, vol. 10, no. 9, pp. 1196-1201, 1992. 
[16] H. Naruse, M. Tateda, H. Ohno, and A. Shimada, "Dependence of the Brillouin gain spectrum on linear strain distribution for optical time-domain reflectometer-type strain sensors," Applied Optics, vol. 41, no. 34, pp. 7212-7217, 2002.

[17] S. Minakuchi, T. Mizutani, H. Tsukamoto, M. Nishio, Y. Okabe, and N. Takeda, "Brillouin spectral response depending on strain non-uniformity within centimeter spatial resolution and its application to internal damage detection in large-scale composite structures," Structural Durability \& Health Monitoring, vol. 4, no. 4, pp. 199-219, 2008.

[18] S. Minakuchi, Y. Okabe, T. Mizutani, and N. Takeda, "Barely visible impact damage detection for composite sandwich structures by optical-fiber-based distributed strain measurement," Smart Materials and Structures, vol. 18, no. 8, pp. 085018, 2009.

[19] K. Kishida, C. H. Li, and K. Nishiguchi, "Pulse pre-pump method for $\mathrm{cm}$-order spatial resolution of BOTDA," in Proc. SPIE, vol. 5855, pp. 559-562, 2005.

[20] Neubrex Co., Ltd., 2013 (available from http://www.neubrex.com/).

[21] D. Zenkert, The handbook of sandwich construction. Warrington: Engineering Materials Advisory Services Limited, 1997.

[22] S. Abrate, Impact on composite structure. Cambridge, UK: Cambridge University Press, 1998.

[23] S. Minakuchi, Y. Okabe, and N. Takeda, "Real-time detection of debonding between honeycomb core and facesheet using a small-diameter FBG sensor embedded in adhesive layer," Journal of Sandwich Structures and Materials, vol. 9, no. 1, pp. 9-33, 2007.

[24] S. Minakuchi, H. Tsukamoto, and N. Takeda, "Detecting water accumulation in honeycomb sandwich structures by optical-fiber-based distributed temperature measurement," Journal of Intelligent Material Systems and Structures, vol. 20, no. 18, pp. 2249-2255, 2009.

[25] Y. Hirose, M. Hojo, A. Fujiyoshi, and G. Matsubara, "Suppression of interfacial crack for foam core sandwich panel with crack arrester," Advanced Composite Materials, vol. 16, no. 1, pp. 11-30, 2007.

[26] S. Minakuchi, I. Yamauchi, N. Takeda, and Y. Hirose, "Memorizing and detecting an arrested crack in a foam-core sandwich structure using embedded plastic materials and fiber-optic sensors," Smart
Materials and Structures, vol. 21, no. 5, pp. 055025, 2012.

[27] R. Gafsi and M. A. El-Sherif, "Analysis of induced-birefringence effects on fiber Bragg gratings," Optical Fiber Technology, vol. 6, no. 3, pp. 299-323, 2000.

[28] A. P. Zhang, B. O. Guan, X. M. Tao, and H. Y. Tam, "Experimental and theoretical analysis of fiber Bragg gratings under lateral compression," Optics Communications, vol. 206, no. 1-3, pp. 81-87, 2002.

[29] S. Minakuchi, I. Yamauchi, N. Takeda, and Y. Hirose, "Detecting an arrested crack in a foam-core sandwich structure using an optical fiber sensor embedded in a crack arrester," Advanced Composite Materials, vol. 20, no. 5, pp. 419-433, 2011.

[30] S. Minakuchi, N. Takeda, S. Takeda, Y. Nagao, A. Franceschettic, and X. Liu, "Life cycle monitoring of large-scale CFRP VARTM structure by fiber-optic-based distributed sensing," Composites Part A: Applied Science and Manufacturing, vol. 42, no. 6, pp. 669-676, 2011.

[31] S. Minakuchi, T. Umehara, K. Takagaki, Y. Ito and N. Takeda, "Life cycle monitoring and advanced quality assurance of L-shaped composite corner part using embedded fiber-optic sensor," Composites Part A: Applied Science and Manufacturing, vol. 48, pp. 153-161, 2013.

[32] K. Takagaki, S. Minakuchi, and N. Takeda, "Fiber-optic-based life cycle monitoring of through-thickness strain in thick CFRP pipes," Journal of the Japan Society for Composite Materials, vol. 39, no. 4, pp. 143-151, 2013.

[33] Y. Ito, S. Minakuchi, T. Mizutani, and N. Takeda, "Cure monitoring of carbon-epoxy composites by optical-fiber-based distributed strain-temperature sensing system," Advanced Composite Materials, vol. 21, no. 3, pp. 259-271, 2012.

[34] R. T. Jones, D. G. Bellemore, T. A. Berkpff, J. S. Sirkis, M. A. Davis, M. A. Putnum, et al., "Determination of cantilever plate shapes using wavelength division multiplexed fiber Bragg grating sensors and a least-squares strain-fitting algorithm," Smart Materials and Structures, vol. 7, no. 2, pp. 178-188, 1998.

[35] C. C. Quach, S. L. Vazquez, A. Tessler, J. P. Moore, E. G. Cooper, and J. L. Spangler, "Structural anomaly detection using fiber optic sensor and inverse finite element method," in AIAA Guidance, Navigation, and Control Conference and Exhibit, San Francisco, CA, Aug. 15-18, pp. 2005-6357, 
2005.

[36] M. Nishio, T. Mizutani, and N. Takeda, "Shape reconstruction of composite structures using high-resolution distributed strain data from Brillouin scattering based optical fiber sensing system," Journal of the Japan Society for Aeronautical and Space Science, vol. 56, no. 658, pp. 522-529, 2008.

[37] M. Nishio, T. Muzutani, and N. Takeda, "Structural shape reconstruction with consideration of the reliability of distributed strain data from a Brillouin-scattering-based optical fiber sensor," Smart Materials and Structures, vol. 19, no. 3, pp. 035011, 2010.

[38] S. Minakuchi, H. Tsukamoto, H. Banshoya, and N. Takeda, "Hierarchical fiber-optic-based sensing system: Impact damage monitoring of large-scale
CFRP structures," Smart Materials and Structures, vol. 20, no. 8, pp. 085029, 2011.

[39] Structural Monitoring Systems Ltd., 2013 (available from: http://www.smsystems.com.au/).

[40] D. P. Barton, "Comparative vacuum monitoring (CVM $\left.{ }^{\mathrm{TM}}\right)$," in Encyclopedia of Structural Health Monitoring. Chichester, UK: Wiley, 2009, pp. 1849-1865.

[41] D. Roach, "Real time crack detection using mountable comparative vacuum monitoring sensors," Smart Structures and Systems, vol. 5, no. 4, pp. 317-328, 2009.

[42] S. Minakuchi, H. Banshoya, S. Ii, and N. Takeda, "Hierarchical fiber-optic delamination detection system for carbon fiber reinforced plastic structures," Smart Materials and Structures, vol. 21, no. 10, pp. 105008, 2012. 